\title{
DIFICULDADES DOS ALUNOS COM OS REGISTROS DE REPRESENTAÇÃO SEMIÓTICA EM AULAS DE FÍSICA DE UMA ESCOLA BRASILEIRA DE ENSINO MÉDIO: O X DA QUESTÃO
}

\author{
DIFFICULTIES OF STUDENTS WITH RECORDS OF SEMIOTIC \\ REPRESENTATION IN PHYSICS CLASSES OF A BRAZILIAN \\ SCHOOL OF SECONDARY EDUCATION: THE X OF THE ISSUE
}

\section{DIFICULTADES DE LOS ALUMNOS CON LOS REGISTROS DE REPRESENTACIÓN SEMIÓTICA EN CLASES DE FÍSICA DE UNA ESCUELA BRASILEÑA DE EDUCACIÓN SECUNDÁRIA:} LA X DEL PROBLEMA

\author{
Maria Guiomar Carneiro Tommasiello ${ }^{\mathrm{I}}$ \\ LuANy Renata dos SANTOS ${ }^{\mathrm{II}}$ \\ SAMARA Dilio Franzol ${ }^{\text {III }}$
}

\begin{abstract}
Resumo Este trabalho é parte de uma pesquisa em andamento com apoio da FAPESP, cujo título é "Pesquisa-Intervenção na Prática Pedagógica de Professores de Ciências da Natureza e Matemática do Ensino Médio de Escolas de Ensino Integral”, em continuidade a outras que têm como foco o ensino de Física em escolas brasileiras de nível médio e o estudo dos registros de representação semiótica utilizados. Em aulas de Física foram observados os registros de representação semiótica mobilizados pelos professores e as suas articulações por meio do tratamento e da conversão. Na conversão de exercícios em língua natural para uma expressão algébrica, os alunos demonstram dificuldade em reconhecer os dados e suas respectivas representações, além de não distinguirem o mesmo objeto matemático, no caso, a incógnita, representada por letras diferentes de X.
\end{abstract}

Palavras-chave: Semiótica; Ensino de física; Ensino médio.

${ }^{\mathrm{I}}$ Universidade Metodista de Piracicaba (UNIMEP), Piracicaba/SP - Brasil "Universidade Metodista de Piracicaba (UNIMEP), Piracicaba/SP - Brasil

IIIUniversidade Metodista de Piracicaba (UNIMEP), Piracicaba/SP - Brasil 
ABstract This work is part of an ongoing research with support from FAPESP whose title is "Research-Intervention in Pedagogical Practice of Teachers of Natural Sciences and Mathematics in the Integral Secondary Education", in continuity with the other, that focus on the teaching of Physics in Brazilian schools of medium level and the study of records of semiotics representation used. In the Physics classes were observed the records of representation semiotics mobilized by the teachers and their joints by means of treatment and conversion. In the conversion of exercises in the natural language to an algebraic expression the students demonstrate difficulty to recognize the data and their respective representations, besides does not recognize the same mathematical object, in this case, the unknown, represented by different letters of X.

Keywords: Semiotic; Teaching of Physics; Middle school.

Resumen Este trabajo es parte de una investigación en curso con el apoyo de FAPESP, titulado "Investigación-Intervención en la práctica docente de Maestros de Ciencias de la Naturaleza y Matemáticas de la escuela secundaria de Escuelas de educación integral" en continuidad con las otras, que se centran en la enseñanza de la física en las escuelas brasileñas de nivel medio y el estudio de los registros de representación la semiótica utilizada. En las clases de Física fueron observados los registros de representación semiótica movilizados por los profesores y sus articulaciones por medio de tratamiento y de la conversión. En la conversión de los ejercicios en lenguaje natural para una expresión algebraica, los estudiantes demuestran dificultades en reconocer los datos y sus respectivas representaciones, y no reconocen el mismo objeto matemático, en este caso, la incógnita, representado por letras diferentes de X.

Palabras claves: Semiótica; Enseñanza de la física; Enseñanza intermedia.

\section{INTRODUÇÃo}

Este trabalho é parte de uma investigação em andamento com o apoio da FAPESP (processo 2015-21973-2), cujo título é "Pesquisa-Intervenção na Prática Pedagógica de Professores de Ciências da Natureza e Matemática do Ensino Médio de Escolas de Ensino Integral"1, em continuidade a outras na mesma linha, cujo foco é o ensino e a aprendizagem de Matemática e Ciências da Natureza em nível médio. Nessa primeira fase do projeto, tem se procurado desenvolver um quadro teórico de análise sob a luz da teoria dos registros de representação semiótica de Duval (2003) para então focalizarmos os registros no ensino de Matemática, Física e Química de uma escola brasileira de ensino integral de nível médio. Neste trabalho, são apresentados alguns dados da área de Física.

1 Apoios: FAPESP - Fundação de Amparo à Pesquisa do Estado de São Paulo e PIBIC/CNPq - Programa Institucional de Bolsas de Iniciação Científica /Conselho Nacional de Desenvolvimento Científico e Tecnológico.

24 Comunicações |Piracicaba | v. 24 | $n .1$ | p. 23-34 | janeiro-abril 2017 
Dados recentes do IDEB, Índice de Desenvolvimento da Educação Básica, apontam o baixo desempenho dos alunos do ensino médio, especialmente em matemática, reforçando a ideia de que é necessária uma reforma do Ensino Médio (BRASIL, 2016). Não saber matemática é um fato que repercute em outras áreas das ciências exatas, que têm essa ciência como linguagem.

Especialistas atribuem o fracasso do ensino médio às fragilidades do currículo, como o excesso de disciplinas obrigatórias que não conversam entre si, à falta de perspectivas dos jovens, entre outras questões (TAKAHASHI, 2015). Para Carneiro (2012), o colapso do ensino médio está na existência do docente leigo/temporário, no desprestígio profissional e social do professor, na dessignificação do ensino médio e nos baixos salários dos docentes.

Com certeza são questões fundamentais, entretanto, temos como pressuposto que o péssimo desempenho dos alunos nas áreas de exatas está fortemente relacionado à linguagem matemática, às representações simbólicas não significadas pelos alunos.

Como os objetos matemáticos não são objetos reais, palpáveis, é preciso dar representantes como um número, um vetor, entre outros. Damm (1999, apud VERTUAN, 2007, p.19) coloca que "[...] não existe conhecimento matemático que possa ser mobilizado por uma pessoa, sem o auxílio de uma representação". Em Matemática, no entanto, é comum se confundir o objeto representado com a representação em si, por isso é importante a utilização no ensino de diferentes representações de um mesmo objeto. Por exemplo, a função polinomial do $2^{\circ}$ grau $\mathrm{y}=\mathrm{ax}^{2}+\mathrm{bx}+\mathrm{c}$ é confundida com o objeto polinomial do $2^{\circ}$ grau, quando é apenas uma das muitas representações desse objeto matemático. (VERTUAN, 2007).

As representações, segundo Duval (2003) podem ser mentais (conjunto de imagens e concepções que um indivíduo pode ter sobre um objeto), internas ou computacionais (caracterizam-se pela execução automática de uma informação) e semióticas (produções constituídas pelo emprego de signos pertencentes a um sistema de representação, como meio de exteriorizar as representações mentais para fins de comunicação). Cabe destacar, no entanto, que para Duval (2012, p.269), as representações semióticas "não são somente necessárias para fins de comunicação, elas são igualmente essenciais para a atividade cognitiva do pensamento".

Os diferentes tipos de sistemas de representação semiótica utilizados em matemática foram designados "registros" e existem, segundo Duval (2003), quatro tipos: os multifuncionais discursivos (língua natural - os tratamentos não são algoritmizáveis) e os não-discursivos (figuras geométricas), e os monofuncionais discursivos (sistemas de escritas: numéricas, algébricas, simbólicas - os tratamentos são principalmente algoritmos) e os não-discursivos (gráficos cartesianos).

De acordo com Duval (2009), para que um sistema de representação semiótica seja considerado um registro de representação semiótica, três atividades cognitivas são necessárias:

1- a formação de uma representação identificável; 2-o tratamento de um registro de representação; 3 - a conversão de um registro de representação.

- Representação identificável: quando é reconhecida pelos sujeitos devido às suas características. Uma poesia é reconhecida como tal entre vários tipos de textos por ser composta por versos contendo métricas, por exemplo. 
- Tratamentos: trata-se de transformações de várias representações no âmbito de um mesmo registro. É uma transformação interna a um registro.

- Conversões: são as transformações de registros, porém se conserva o mesmo objeto. A conversão de uma escrita algébrica de uma equação em sua representação gráfica, por exemplo. A conversão é uma transformação externa ao registro de partida.

A essência da atividade matemática fundamenta-se na mobilização simultânea de ao menos dois registros de representação ao mesmo tempo. Ou em outras palavras, "a compreensão da matemática implica a capacidade de mudar de registro" (DUVAL, 2003, p. 21).

Quais poderiam ser essas conversões? García e Palacios (2006) citam possíveis conversões entre os registros de representação semiótica: enunciado - equação; equação - enunciado; gráfico cartesiano - equação; equação - gráfico cartesiano; gráfico cartesiano - enunciado; enunciado - gráfico cartesiano; tabela de dados - gráfico cartesiano. Em geral, segundo os autores, as conversões que envolvem gráficos cartesianos são as mais problemáticas.

Por sua vez, Hillesheim e Moretti (2013) consideram que a conversão não desperta tanto a atenção dos educadores, talvez por não ter um papel de prova ou justificação nos processos matemáticos.

Mesmo sem ter clareza do papel dos registros de representação semiótica no ensino-aprendizagem de conceitos da área de ciências exatas, é possível que os professores tenham já verificado que em determinadas situações de conversão, os alunos têm mais facilidade em reconhecer o objeto matemático e em outras, não. Quando há relação entre a frase e a expressão algébrica, Duval (2009) considera que há congruência.

São três os critérios que Duval (2009) utiliza para considerar se há ou não congruência entre duas representações semióticas: correspondência semântica dos elementos significantes, univocidade semântica terminal e correspondência na ordem da organização das unidades compondo cada uma das duas representações.

Semmer, Silva e Neves (2014) exemplificam a congruência com a seguinte situação: o triplo de um número acrescido de duas unidades é igual a 17. A linguagem algébrica será $3 x$ $+2=17$. Têm-se dois signos quando anuncio que o triplo de um número corresponde a $3 \mathrm{x}$ : o 3 representa o triplo e a quantidade três, além do x que representa o número desconhecido. Ao fazer a conversão de uma linguagem à outra, organiza-se na mesma ordem, ou seja, realiza-se a leitura da língua natural da esquerda para a direita. E a univocidade semântica terminal acontece quando, numa conversão, a informação é mantida. No exemplo dado (3x $+2=17$ ), a operação semântica indicava a operação multiplicativa e essa operação continua mesmo tendo sido mudado o registro de representação semiótica.

Apesar de os registros de representação semiótica serem mais explorados por pesquisadores do campo da matemática, as atividades na área de física são também caracterizadas pela dependência desses registros de representação, bem como, pela grande variedade desses registros. Vários autores já se debruçaram sobre os registros de representação semiótica em física, como Camargo Filho (2011), com gráficos cartesianos de cinemática; Santos e Curi (2012) com funções horárias de cinemática; Flores e Moretti (2008) com congruência semântica, entre outros. 
Pelo exposto, este trabalho tem como objetivo geral identificar e analisar os registros de representação semiótica utilizados pelos professores em sala de aula e possíveis dificuldades dos alunos com operações de tratamento e de conversão realizadas, de forma a contribuir para a melhoria do ensino/aprendizagem de física do ensino médio.

\section{Problema de inVestigação}

Considerando a importância dos registros de representação semiótica na aprendizagem das ciências da natureza e as conversões entre estes, o problema de investigação pode ser assim enunciado: A forma como os professores lidam com os registros de representação semiótica em aulas do ensino médio e com as conversões entre estes possibilita uma aprendizagem efetiva de conceitos científicos de Física? São as seguintes questões de pesquisa: Quais os registros de representação semiótica mobilizados pelos professores de física em aulas de mecânica e calorimetria? Como os docentes articulam esses registros por meio do tratamento e da conversão? Quais as dificuldades de aprendizagem?

\section{Metodologia}

Trata-se de uma pesquisa de natureza qualitativa com o objetivo de obtenção de dados descritivos acerca do cotidiano da sala de aula, obtidos por meio de contatos diretos entre o pesquisador e os pesquisados- docente e alunos. O estudo qualitativo é rico em dados descritivos, tem um plano aberto e flexível e focaliza a realidade de forma complexa e contextualizada. (LUDKE; ANDRÉ, 1986).

O estudo foi realizado com aulas de um banco de dados, constituído por filmagens já realizadas em pesquisas anteriores em duas escolas estaduais públicas estaduais de ensino médio situadas em duas cidades vizinhas do interior do Estado de São Paulo. Um dos professores $(\mathrm{P} 1)$ é engenheiro agrônomo, com Licenciatura em Biologia e com curso de complementação em Física e o outro docente (P2) graduou-se em Licenciatura em Física e tem curso de especialização em Física para o ensino médio. As turmas eram relativamente pequenas, em torno de 25 alunos. A idade média dos alunos da primeira série era de 15 a16 anos e da segunda série, de 16 a 17 anos.

Para responder às questões do estudo foram observadas algumas aulas nas duas séries, em especial as que envolvessem resolução de exercícios, pois nestas, a participação dos alunos é, em geral, maior do que em aulas essencialmente teóricas. As aulas foram transcritas e escolhidos episódios que envolvessem dúvidas dos alunos com a conversão entre diferentes registros de representação semiótica.

\section{Análise dos EPISÓDIOS}

\section{$1^{0}$ Episódio}

Em uma aula de segunda série do ensino médio sobre calorimetria, o professor (P1), ao resolver um exercício, chama a atenção dos alunos para o significado do símbolo delta na fórmula de calor. 
O professor lê um exercício de uma lista disponibilizada aos alunos sobre uma mistura de água morna com água quente: "Qual a temperatura de equilíbrio térmico quando, em um calorímetro ideal, misturamos $200 \mathrm{~g}$ de água a $20^{\circ} \mathrm{C}$ com $400 \mathrm{~g}$ de água a $80^{\circ} \mathrm{C}$ ?"

1-Professor: (...) Atenção! Prestem a atenção agora. Qual é a fórmula de DELTA $Q^{2}$ mesmo? Calor...?

2-Aluna1: Massa vezes ...??

3-Professor: vezes Calor especifico vezes variação de temperatura. Q é dado em caloria, $m$ em gramas, Calor especifico lá e DELTA TETA em ${ }^{\circ} C$. [...] E porque eu coloco um DELTA na frente? O que significa DELTA para vocês?

4-Alunos: Supermercado??!! [na cidade há uma rede de supermercados com esse nome]

5-Professor: O quê? Supermercado? Que mais? Aquela construtora lá né?! ... DELTA S, DELTA T, DELTA V, DELTA...

6-Aluna 1: DELTA é uma fórmula!!

7-Professor: DELTA é a diferença! Sempre DELTA é o final menos o ...?? O que é DELTA TETA pessoal? Diferença de temperatura. Temperatura final menos temperatura inicial. [...] Deixa eu perguntar uma coisa, essa água 1, ela ganhou Calor ou ela perdeu Calor?

8-(...) Os alunos ficam em silêncio.

9-O professor começa a fazer as substituições na fórmula (massa de água 1, calor específico e variação da temperatura-temperatura de equilibrio térmico menos a temperatura inicial). Ao chegar à temperatura de equilíbrio térmico, o professor diz: quanto vale? Eu não tenho. Se eu não tenho, como eu a chamo?

10-Aluno 2: Ponto de interrogação!!??

11-Alunos (em coro): X!!!

12- Professor: X???

13-Alunos: Pode ser!

14- Professor: Não!!! X é na Matemática, aqui nossa incógnita tem nome, temperatura... final. Então a gente vai pôr o ideal, TETA $(\boldsymbol{\Theta})$.

[O professor continua fazendo as substituições. (...)]

15-Professor: Bom! Prestem a atenção depois vocês copiam. Vamos lá! ... É isso que vocês iam fazer, lógico, só estou fazendo para vocês.

$200 x 1 \times(\boldsymbol{\Theta}-20)+400 \times 1 \times(\boldsymbol{\Theta}-80)=0$

$200 x(\boldsymbol{\Theta}-20)+400 x(\boldsymbol{\Theta}-80)=0$

16-E agora, o que eu faço? Pergunta o professor dirigindo-se à classe (...)

17-Aluna 1: Tem que fazer 200 vezes ZERO?? Pergunta a aluna [provavelmente ela quis dizer 200 vezes TETA, cujo símbolo é $\Theta$, parecido com 0]

18-O professor não se dá conta do que diz a aluna. Dirige-se para a classe: Tem que fazer "chuveirinho" aqui. Distributiva!

19-O professor pede novamente para os alunos terminarem o cálculo e diz, olha, TETA é X!! Se fosse X vocês fariam! Só porque eu passei para TETA vocês não sabem??

${ }_{2}$ A equação é dada por $\mathrm{Q}=\mathrm{mc} \Delta \boldsymbol{\Theta}$ e não por $\Delta \mathrm{Q}$ (delta $\mathrm{Q}$ ), pois $\mathrm{Q}$ é quantidade de calor em trânsito e não uma variação de calor.

28 Comunicações |Piracicaba | v. 24 | 1.1 | p. 23-34 |janeiro-abril 2017 
[O professor coloca o resultado no quadro $\Theta=60^{\circ} \mathrm{C}$, mas não faz comentários sobre o valor, não volta à pergunta do problema de forma a explicitar o que estavam buscando].

Análise: Nesse episódio são utilizados dois registros de representação semiótica: a língua natural (registro multifuncional discursivo) e equações algébricas (registro monofuncional discursivo). Observam-se primeiramente as dificuldades dos alunos em fazer a conversão, em relacionar as grandezas dadas em linguagem natural com os seus representantes na equação algébrica, em especial, a temperatura que tem como representante a letra grega TETA $(\boldsymbol{\Theta})$. Outra dificuldade é que o exercício não apresenta uma congruência semântica sendo que a incógnita aparece nos dois termos da equação algébrica. Ou seja, é preciso a realização de um tratamento, de operações no âmbito do mesmo registro, para evidenciar a incógnita. Mesmo envolvendo uma propriedade estudada em matemática-que é a propriedade distributiva - os alunos têm problemas com a resolução da equação e cálculo da temperatura de equilíbrio térmico, não identificada como incógnita, uma vez que usa como representante a letra TETA $(\boldsymbol{\Theta})$ e não a letra X. Além da letra TETA não ser significativa como representante da temperatura, esta pode ter sido confundida com o número zero, pelas características visuais próximas.

\section{$2^{\circ}$ EpISÓdio}

Em uma aula sobre conservação da energia Mecânica de $1^{\mathrm{a}}$ série do ensino médio, o professor (P1) retoma um exercício colocando na lousa a equação de energia mecânica. Um corpo cai de determinada altura e se deseja saber a velocidade que chega ao solo.

1-Professor: (...) quando chegar aqui em baixo, prestes a se chocar com o solo, eu não tenho mais o quê??

2-Aluno 2: Velocidade!!

3-Professor: Não!! Altura!!! Não tenho mais altura, se eu não tenho mais altura, ó $h$-o professor lê o que havia escrito na lousa - no momento de choque com o solo teremos só energia cinética, a energia potencial valerá zero porque $h=0$. Então no momento do choque eu posso dizer o quê? ... [O professor para e faz uma anotação na lousa].

4-Aluna 3: O que quer dizer $h$ ?

5-Professor: Altura. [...] a gente vê isso desde trigonometria, que você tem o triangulo e pergunta-se qual é a altura do triângulo?! Que letra que vocês usam? h. A letra a no triângulo é o quê?

6-Aluno 4: a no triângulo??

7-Professor: $a, b$, c, triângulo retângulo? Quadrado da hipotenusa igual à soma $a^{2}=b^{2}+c^{2}$.

8-Aluna 3: Então, mas será que usa h? Imagina!

9-Professor: Mas o hé a altura. O que é a altura? A altura é um segmento que vai de um dos vértices do triângulo até o outro lado, perpendicularmente a ele.

10-Aluna 3: Ah?? [ Faz uma expressão como se estivesse confusa] 
11-Professor: $E m=E c+E p$ e se no momento do choque a energia potencial vale zero, o que nós temos? Em $=E c=1.500 \mathrm{~J}$. Bom, então eu posso dizer o quê? Vamos fazer a fórmula antes...

$$
E c=m_{x} v^{2} / 2
$$

$1.500=$ quanto vale a massa? $3_{x}$ quanto vale a velocidade? Não sei dividido por $2 !$

$$
1.500=3 v_{x} v^{2} / 2 \text {. }
$$

Agora vocês podem continuar. (...) Quem pode me dar essa solução?...

12-Aluna 1: Professor, eu não tenho o valor do V, posso SUBSTITUIR por 1??

13-Professor, NÃO!! Vé a incógnita!!!!É o X da questão!!

Análise: Nesse episódio também são utilizados dois registros de representação semiótica: a língua natural (registro multifuncional discursivo) e equações algébricas (registro monofuncional discursivo). Há dificuldades na conversão, em relacionar as grandezas dadas em linguagem natural com os seus representantes na equação algébrica. Uma aluna questiona o símbolo h, que representa a altura. As explicações do professor sobre o significado da grandeza $\mathrm{h}$ também não ajudaram a dirimir as dúvidas dos alunos. A pergunta da aluna, quanto à possibilidade de substituição da velocidade por 1 , mostrou que a velocidade não foi identificada como sendo a incógnita. Na mesma linha de Rosa (2008), observa-se que os alunos têm dificuldades com a interpretação de textos e em perceber como a linguagem matemática pode "substituir" o texto em língua natural.

\section{$3^{0}$ EPISÓdIO}

Este episódio é um recorte de uma aula de $1^{\circ}$ ano intitulada "Trabalho da força de atrito e a variação da Energia Cinética".

O professor (P2) coloca no quadro o título citado e as seguintes equações:

Força de atrito $->F a t=u \cdot N$; Trabalho da força de atrito $->u \cdot N \cdot d$ e Energia Cinética (variação) -> $E p \quad E i$

Professor: Essa igualdade $T f a t=\Delta E$, que nós já vimos, vamos utilizar para encontrar a relação entre a energia cinética e o trabalho da força de atrito.

[O professor faz a substituição de cada lado da igualdade, e obtém o seguinte:]

$$
u \cdot m \cdot g \cdot d=\frac{m \cdot V^{2}}{2}
$$

[Ele pergunta aos alunos o que há em comum nos dois lados da igualdade, mas como não obtém resposta alguma, ele faz um corte nos dois $m$ (cancelando as massas) e apresenta a seguinte relação:

$$
u \cdot g \cdot d=\frac{V^{2}}{2}
$$

30 Comunicações |Piracicaba | v. 24 | 1.1 | p. 23-34 | janeiro-abril 2017 
[Logo a seguir, utiliza o caderno do aluno para a resolução de um exercício que faz uso da fórmula encontrada. $\mathrm{O}$ exercício consistia em encontrar a distância de frenagem de determinado veículo com uma velocidade inicial de $45 \mathrm{~km} / \mathrm{h}$ até parar, sabendo-se que o coeficiente de atrito é 0,8 . O professor alerta que na equação de energia cinética a velocidade precisa ser em $\mathrm{m} / \mathrm{s}$.] [...]

$$
\begin{aligned}
& 0,8 \cdot 10 \cdot d=\frac{12,5^{2}}{2} \\
& 8 d=\frac{156,25}{2} \\
& 8 d=78,14 \\
& d=\frac{78,14}{8} \\
& d=9,8 m
\end{aligned}
$$

Professor: Pronto!! d, que era a nossa incógnita, é igual a 9,8 m.

[Alguns alunos perguntam em conjunto, após ouvirem a palavra incógnita]

Alunos: Se era incógnita, por que não usou X? Não posso usar X em vez de d?

Análise: Os dois registros de representação semiótica utilizados são: a língua natural (registro multifuncional discursivo) e equações algébricas (registro monofuncional discursivo), havendo conversão de um registro para o outro. Como o professor sinaliza a equação algébrica a ser empregada, e ele próprio faz as substituições dos valores das grandezas na equação e o cálculo do valor da distância, não surgem questionamentos por parte dos alunos sobre a conversão e acerca do tratamento. Aparentemente, o exercício era de fácil solução, ou seja, a conversão parecia próxima de uma situação de simples codificação (quando o aluno é capaz de retirar do texto a informação que necessita), mas a pergunta dos alunos surpreende, pois eles não tinham dado significado ao $\underline{\mathrm{d}}$ da equação, como sendo a incógnita. Possivelmente, a equação simplificada pelo professor não fazia sentido a esses discentes.

\section{DisCUSSÃo}

O uso de Registros de Representação Semiótica é recorrente nas aulas de física observadas e entre esses registros são privilegiadas pelo professor a língua natural (registro multifuncional discursivo) e equações algébricas (registro monofuncional discursivo). Nos três episódios, houve a conversão de um registro para o outro, sempre neste sentido: língua natural- equação algébrica.

Nos três episódios não se percebe um esforço do professor em deixar claro aos alunos as relações entre as grandezas dadas em linguagem natural e seus representantes nas equações algébricas, com destaque para a grandeza que se deseja buscar, a incógnita. Polya (1995, apud PEDUZZI, 1997, p.232) levanta essa questão quando considera que o primeiro 
passo para a resolução do problema é compreendê-lo: reunir informações sobre o problema e a respeito da pergunta: O que se quer, o que é desconhecido? O que se tem, quais são os dados e as condições?

Com a fala do turno 19 do episódio 1, o professor mostra que tem clareza da dificuldade dos alunos com as incógnitas, mas talvez não tenha a dimensão do custo cognitivo para o aluno em dar significados às letras utilizadas como representantes das grandezas físicas. Apesar da boa formação dos professores na área de física, possivelmente eles desconhecem a dificuldade dos discentes com a conversão entre os registros, justificada por não ser uma atividade natural a ser realizada pelos alunos, tendo que ser trabalhada em sala de aula. De acordo com Duval (2009, p.63), "a conversão das representações semióticas constitui a atividade cognitiva menos espontânea e mais difícil de adquirir para a grande maioria dos alunos".

Os professores também não retomam a questão inicial de forma a dar sentido para a resposta numérica. Ou seja, depois de realizados os cálculos, poderiam retornar à língua natural, fazendo o sentido inverso do inicial. Polya (1995, apud PEDUZZI, 1997) considera que o retrospecto da resolução completa pode consolidar o conhecimento e aperfeiçoar a capacidade dos alunos em resolver problemas.

\section{CONSIDERAÇõES FINAIS}

Em resposta às questões de pesquisa, conclui-se que os registros de representação semiótica privilegiados pelos dois professores são os multifuncionais e monofuncionais discursivos (língua natural, enunciados, equações algébricas) e a conversão se dá do registro língua natural para o registro algébrico. Os alunos mostram dificuldades em identificar as grandezas físicas em língua natural com as suas respectivas representações nas equações algébricas; dificuldades com as operações de tratamento e problemas de inter-relações entre conhecimentos de matemática e de física.

Os dados sinalizam que os discentes têm dificuldade em aceitar outro signo representando a incógnita, que não seja a letra X. Ou seja, confundem o objeto matemático com a sua representação semiótica. Para Duval (2009), essa confusão faz que os conhecimentos adquiridos tornem-se rapidamente inutilizáveis fora de seus contextos de aprendizagem.

É fundamental o professor proporcionar situações que envolvam também a passagem de ida e vinda entre diferentes registros para que o aluno possa visualizar um mesmo objeto matemático sob diferentes formas, para evitar, segundo Duval (1993, apud COLOMBO et al, 2009, p.102), que se forme um "enclausuramento de registros" que leva o indivíduo a "ver" um objeto matemático de apenas uma maneira e não conseguir pensar de forma diferente.

Concordamos com Vertuan (2007, p.20), quando considera que o modo como o aluno lida com uma representação semiótica "revela de alguma forma como ele representou essa informação internamente. Saber interpretar a representação produzida pelo aluno pode ajudar o professor a realizar intervenções mais adequadas no seu processo de construção do conhecimento". 


\section{REFERÊNCIAS}

BRASIL. Instituto Nacional de Estudos e Pesquisas Educacionais Anísio Teixeira. IDEB. Índice de Desenvolvimento da Educação Básica. Disponível em: $<$ http://portal.inep.gov. br/visualizar/-/asset_publisher/6AhJ/content/dados-do-ideb-2015-ja-estao-disponiveis-para-consulta $>$ Acesso em 15 de setembro de 2016.

CAMARGO FILHO, P. S. de. Dificuldades semióticas na construção de gráficos cartesianos em cinemática. Dissertação de Mestrado, Curso de Mestrado em Ensino de Ciências e Educação Matemática, Universidade Estadual de Londrina, Londrina/PR/Brasil, 2011.

CARNEIRO, M.A. O Nó do Ensino Médio. Petrópolis, RJ: Vozes, 2012.

COLOMBO, J. A. A, BUEHRING, R. S.; MORETTI, M.T. Registros de representação semiótica, tarefas e análise de dados: articulações em torno do currículo de matemática. Revista Eletrônica de Educação Matemática. 4 (8), 90-113, 2009.

DUVAL, R. Registros de representação semiótica e funcionamento cognitivo da compreensão em matemática. In: Machado, S. D.A. (Org.). Aprendizagem em matemática: registros de representação semiótica. Campinas/SP: Papirus, p. 11-33, 2003.

DUVAL, R. Semiósis e pensamento humano: registros semióticos e aprendizagens intelectuais. São Paulo: Editora Livraria da Física, 2009.

DUVAL, R. Diferenças semânticas e coerência matemática: introdução aos problemas de congruência. Tradução: Méricles Thadeu Moretti. Revista Eletrônica de Educação Matemática. Florianópolis, SC, 7, n. 1, 2012.

FLORES, C. R.; MORETTI, M. T. A articulação de registros semióticos para a aprendizagem: analisando a noção de congruência semântica na Matemática e na Física. Perspectivas da educação matemática. Campo Grande: MS, 1 (1), 25-40, 2008.

GARCÍA, J. J.; PALACIOS, F. J. P. Cómo usan los profesores de Química las representaciones semióticas? Revista eletrônica de Enseñanza de las Ciencias, 5 (2), 247-259, 2006.

HILLESHEIM, S. F.; MORETTI, M.T. Alguns aspectos da noção da congruência semântica presentes no ensino dos números inteiros relativos. Passo Fundo: Espaço Pedagógico, 20 (1), 119-135, 2013.

LÜDKE, M.; ANDRÉ, M.E.D.A. Pesquisa em Educação: abordagens qualitativas. São Paulo/SP: EPU, 1986.

PEDUZZI, L.O.Q. Sobre a resolução de problemas no ensino da física. Caderno Catarinense de Ensino de Física, 14 (3), 229-253, 1997. 
ROSA, C. C. da. Um estudo do fenômeno de congruência em conversões que emergem em atividades de Modelagem Matemática no Ensino Médio. Dissertação de Mestrado, Pós-Graduação em Ensino de Ciências e Educação Matemática, Universidade Estadual de Londrina, Londrina/PR/Brasil, 2008.

SEMMER, S, SILVA, S. De C. R. da; NEVES, M. C. D. Arte e matemática: teoria de registros de representação semiótica e proposta triangular. Revista Ciências \& Ideias, 5 (2), 19-34, 2014.

SANTOS, C.A.B. dos; CURI, E. Registros de representação semiótica e suas contribuições para o ensino de física. Ensaio Pesquisa em Educação em Ciências, 14 (3), 8595, 2012.

TAKAHASHI, F. Como explicar a baixa qualidade do ensino médio? Folha de São Paulo, São Paulo, 5 set. Caderno Especial- Gestão Escolar, p.2, 2015.

VERTUAN, E. F. Um olhar sobre a modelagem matemática à luz da teoria dos registros de representações semióticas. Dissertação de Mestrado em Ensino de Ciências e Educação Matemática, Universidade Estadual de Londrina/PR/Brasil, 2007.

\section{Dados das Autoras}

\section{Maria Guiomar Carneiro Tommasiello}

Doutora em Tecnologia Nuclear pela Universidade de São Paulo. Professora, pesquisadora e orientadora no Programa de Pós-Graduação em Educação da Universidade Metodista de Piracicaba. Piracicaba/SP - Brasil.mgtomaze@unimep.br

\section{Luany Renata dos Santos}

Discente do Curso de Matemática-licenciatura na Universidade Metodista de Piracicaba. Bolsista PIBIC/CNPQ/UNIMEP. Piracicaba/SP - Brasil. luany.renata@bol.com.br

\section{SAMARA Dilio Franzol}

Graduada em Química-licenciatura pela Universidade Metodista de Piracicaba. Professora de Química da rede pública estadual. Piracicaba/SP - Brasil.samara_franzol@hotmail.com

Submetido em: 29-12-2016

Aceito em: 2-3-2017 